\title{
Pharmacy students' perceived professionalism and application of bioethical principles: Implications for teaching pharmacy ethics for patient-centred pharmacy practice
}

\author{
Roland N Okoro ${ }^{1}$ \& Aminu A Biambo ${ }^{2}$ \\ ${ }^{1}$ University of Maiduguri, Nigeria ${ }^{2}$ Usmanu Danfodiyo University, Nigeria
}

\section{Keywords \\ Professionalism \\ Ethical Dilemmas \\ Bioethics Principles \\ Final-year Pharmacy Students \\ Nigeria}

\section{Correspondence}

Roland N. Okoro

Department of Clinical Pharmacy

and Pharmacy Administration

Faculty of Pharmacy

University of Maiduguri

Borno State

Nigeria

orolandn@gmail.com

\begin{abstract}
Context: Pharmacists providing pharmaceutical care are often confronted with a variety of professional issues, ethical dilemmas and moral problems that require professionalism to appropriately address. Aims: To investigate undergraduate fifthyear (final-year) Bachelor of Pharmacy (B. Pharm.) students' views on professionalism, ethical dilemmas, and their application of bioethical principles, and to compare responses of various groups (pharmacy schools, age, gender, and religion). Methods: This was a cross-sectional survey of the final-year B.Pharm. students in two pharmacy schools in northern Nigeria. The descriptive and inferential statistics (Chi-square or Fisher's exact tests and Independent-sample $t$-test) were utilised to analyse the study data. Statistical significance was considered at $p<0.05$. Results: Ninety-five students, corresponding to $49.5 \%$ response rate, participated in the study. More than $79 \%$ of the students strongly agreed that their actions as future pharmacists could affect how the profession is viewed by patients and other healthcare providers. Patients' right to autonomy and non-maleficence were the most utilised bioethical principles by the participants. Conclusions: The study shows that the participants had good overall perceived professionalism and fair bioethical reasoning. Different bioethical principles were used by various groups considered to support how they would deal with each ethical dilemma. New strategies in teaching pharmacy practice courses by pharmacy educators in these schools are necessary to improve students' bioethical reasoning which will maximise the quality of their future practice.
\end{abstract}

\section{Introduction}

Contemporary pharmacy practice has shifted towards a more patient-centred model of practice due to increased hospitalisations and mortality and associated cost resulting from inappropriate use of medications (Johnson \& Bootman, 1995; Johnson \& Bootman, 1997). Pharmaceutical care reflects this shift and it requires partly, the establishment of an ethical covenant between the pharmacist, the patient and other healthcare practitioners (Kruijtbosch et al., 2018). Many ethical issues arise from the traditional model of pharmacy practice, such as dispensing prescriptions when prescribers fail to completely prescribe; failure to meet legal requirements on the prescription yet the patient needs the medicine; prescribing unlicensed medicines/off-licensed medicine/ those considered less appropriate for prescribing among others. Other extended roles, including pharmaceutical care, have evolved over time. Pharmaceutical care has resulted in an increase in the number and variety of ethical dilemmas and moral problems that pharmacists 
face in their daily practices (Hibbert et al., 2000; Latif, 2001; Wingfield et al., 2004; Kalvemark et al., 2004; Chaar et al., 2005; Cooper et al., 2007; Cooper et al., 2008; Benson et al., 2009).

Ethical dilemmas occur once there is discord on moral behaviours or the application of ethical principles. In pharmacy practice, they consist of diverse scenarios encountered by pharmacists in their daily work in which there are two or several competing ethical principles. They can manifest among pharmacists and with others (clients and physicians) due to differences in the values, sense of justice and fairness of each party (Kruijtbosch et al., 2018). Examples of areas in which such dilemmas may develop include those associated with monitoring and screening of patients with chronic diseases, decisionmaking on drug therapies, providing drug information, referral practices and clinical investigations, among others. Disappointingly, many pharmacists are either unable to detect ethical problems or do not act to address them appropriately (Tekiner, 2017).

Pharmacy practice and education in Nigeria is experiencing many changes (Erah, 2011; Ogaji \& Ojabo, 2014; Ikhile \& Chijioke-Nwauche, 2016). Through the emergence of pharmaceutical care and multidisciplinary drug therapy management globally, pharmacy education in the country has undergone a mild change from a science-based curriculum to a science and practice mixed curriculum. The Bachelor of Pharmacy (B. Pharm.). syllabi of the clinical pharmacy and pharmacy practice courses, including pharmacy ethics and professionalism are still far from adequate (Fakeye et al., 2017). Additionally, hospital pharmacists in the country have commenced on low scale patient-centred care with other healthcare practitioners as a team. This patient-centred pharmacy practice calls for a refocusing on professionalism, ethical dimensions of the pharmacist's position and on the ethical education of pharmacy students to adequately equip them for this task (Ibrahim \& Sharif, 2018).

Professionalism and ethics for pharmacy practice as a component of the undergraduate pharmacy curriculum helps to prepare future pharmacists to begin their professional careers with adequate professional and ethical knowledge, skills and experience in patientcentred care responsibilities (Ibrahim \& Sharif, 2018). Therefore, pharmacy students need to be well-oriented with professional behaviours, ethical issues and ways of implementing the best ethical solution at all times. Knowledge about ethical principles and competence to apply professional judgment is paramount (Sharif et al., 2011).
As healthcare professionals, pharmacists are confronted with critical thinking and decision-making and have to follow ethical guidelines (Sharif et al., 2011). However, the main bioethics principles (autonomy, beneficence, nonmaleficence, and justice) (Warren, 2011) have had a tremendous effect on codes of ethics and the teaching of professional ethics and have become familiar concepts among healthcare practitioners globally (Slomka et al., 2008; Kangasniemi et al., 2015). Nevertheless, there are other ethical principles that could be applied to many healthcare scenarios; therefore, these four principles are not considered absolutes but serve as practice guides (McCormick, 2018).

Through ethical reasoning, pharmacists need to select their best course of action from many, justify and defend it. To be able to do this, the pharmacist must have sound knowledge of the ethical principles and values of the profession (Beauchamp \& Childress, 2001), the legal framework within which the pharmacy profession operates, and the code of ethics of the pharmacy profession (University of Greenwich, 2015). The increasingly important ethical dimension of pharmacy practice and the decreasing interest in this dimension, as it is reflected by the lack of attention given to it, has grave consequences for the profession. Pharmacists just like physicians are expected to practice in an ethically and legally acceptable manner (Vivian, 2010). The emergence of ethical issues due to a lack of internal control could result in liabilities to pharmacists (Buchanan, 2017).

In Nigeria, most of the pharmacy schools currently offer a five-year B.Pharm. programme until it is completely phased out and replaced by the newly approved six-year Doctor of Pharmacy (Pharm.D.) programme (Ogaji \& Ojabo, 2014). The Nigerian B.Pharm. students undertake a mandatory pharmacy law and ethics course with elements of professionalism in the fourth-year (penultimate year) of the programme. The pass mark for this course is $60 \%$. In addition, they participate in a mandatory six months (three months each at the end of the third-year and fourth-year holidays, respectively) experiential-learning programme (Students Industrial Work Experience Scheme [SIWES]) in pharmaceutical industries, community pharmacies, and hospital pharmacies prior to their finalyear (Abdu-aguye et al., 2019). This externship programme affords them the opportunities to apply learning about pharmacotherapeutics, and pharmacy law and ethics in a real-world practice situation, in preparation for the hospital-based pharmacy clerkship and ward round programme which takes place during the last semester of their final-year. 
Studies have considered students' ethical frames of reference (Latif, 2003; Latif, 2004; Cooper et al., 2007; Gallagher, 2011; Okoye et al., 2017; Hanna et al., 2017), including studies that investigated the use of case scenarios to elicit students' responses about how they deal with ethical dilemmas (Donaldson et al., 2010; Frunză, 2011; Schlesselman, 2014; Lindsey \& Olin, 2014; Smith \& Dinkins, 2015; Henning et al., 2016). To the authors' knowledge, no such studies have been conducted among pharmacy students in Nigeria. The authors embarked on this study to further contribute to this area of research by providing baseline data from Nigeria, a subSaharan African country.

This study was aimed to investigate undergraduate finalyear pharmacy students' views on professionalism, ethical dilemmas, and their application of bioethical principles, and to compare responses across various groups (pharmacy schools, age, gender, and religion). The findings of this study may provide insights into the way these students may behave and respond when exposed to ethical dilemmas in practice, both as an intern and practicing pharmacist upon graduation.

\section{Methods}

\section{Design and settings}

This questionnaire-based survey was carried out in August 2018. The University of Maiduguri (UM) and Usmanu Danfodiyo University (UDU) were the study settings. These Universities were purposively selected among the eight universities offering the Pharmacy programme in northern Nigeria. They were chosen because they were established close to each other compared to the rest; therefore, they are expected to have similar infrastructural, student and staff strengths. The UM had a total of 606 registered pharmacy students during the 2018 academic year. Of this number, 106 were in the final-year. Similarly, the UDU had a total of 465 registered pharmacy students, out of which 86 were in the final-year in the 2018 academic year.

\section{Participants and sampling}

In each of the study sites, pharmacy students in the finalyear who had undertaken the pharmacy law and ethics course, as prescribed by the Pharmacists Council of Nigeria, in the fourth-year of their five-year B.Pharm. programme were the study population. These students were invited to participate in the study by their class representative in both Universities. Those who agreed to participate in the study and gave their verbal consent completed the self-administered paper-based questionnaire anonymously during class time. Participants who completed the questionnaire willingly returned them to a research assistant. It took approximately 15 minutes to complete the questionnaire and no incentives were offered to the participants.

\section{Ethical consideration}

The study proposal was reviewed and approval granted by the Faculty of Pharmacy Research Review Board of the University of Maiduguri, Nigeria. Participants gave verbal informed consent prior to questionnaire administration.

\section{Study instrument}

The questionnaire used for this study (Appendix A) was adapted from two previous studies (Schlesselman, 2014; Smith \& Dinkins, 2015) that had proven them robust for baseline studies. This instrument consists of three sections. The first section obtained the social and demographic information (university, age, gender, religion, and tribe) of the participants. The second section was an eight-item professionalism in pharmacy practice (PPP) instrument adapted from Smith \& Dinkins (2015). The third section was the pharmacy ethical dilemmas survey (PEDS) instrument adapted from Schlesselman (2014). The PEDS instrument evaluates participants' actions, choices and the ethical principles that most influenced their decisions (Schlesselman, 2014). The dilemmas associated with sedation, placebo medication, euthanasia (assisted dying) and expired medications were considered. These scenarios evaluated each participant's willingness to dispense the medication when confronted with such dilemmas and the reasons for his/her decision. The PEDS case scenarios were used because pharmacists working in community pharmacies, hospitals, and nongovernmental organisations in Nigeria are also exposed to these dilemmas in practice.

\section{Data collection}

The information collected included sociodemographic characteristics of the study population, their perceptions on professionalism and ethical dilemma.

\section{Data analysis}

Individual items on the PPP items were on the original Likert scale of 1-4, with 1 being strongly disagree, and 4 being strongly agree. For the application of bioethical principles of the PEDS, the 'valid' reason for choosing to 
dispense the medication or not was based on personal views. Data were summarised initially using descriptive statistics (frequency, percentage, and mean). The Chisquare test or Fisher's exact test (for $n<5$ per cell) was used to identify statistically significant difference. Independent-samples $t$-test was used to compare means of two groups (pharmacy schools [UM versus UDU], age [ $\leq 25$ years versus $>25$ years], gender [female versus male], and religion [Islam versus Christianity]); and $p<0.05$ was statistically significant. The IBM Statistical Package for Social Sciences (SPSS) Statistics for Windows, Version 21.0. Armonk, NY: IBM Corp was used for statistical analyses.

\section{Results}

The response rates varied from $39.5 \%(34 / 86)$ at UDU to $57.6 \%(61 / 106)$ at UM with an overall response rate of $49.5 \%$. The majority of the study populations (54.7\%) were males and $68.4 \%$ of them aged 25 years or less. Islamic religion adherents were more dominant in the UDU (88.2\%) compared to UM (67.2\%) with a significant difference $(p=0.041)$. The detailed socio-demographic information of the participants is shown in Table I.

Table I: Demographic characteristics of the participants $(\mathrm{N}=95)$

\begin{tabular}{|c|c|c|c|c|c|}
\hline \multirow[t]{2}{*}{ Variable } & \multicolumn{2}{|c|}{ University } & \multirow[t]{2}{*}{ Total n (\%) } & \multirow[t]{2}{*}{$x^{2}$} & \multirow[t]{2}{*}{$p$ value } \\
\hline & UM n (\%) & UDU n (\%) & & & \\
\hline \multicolumn{6}{|l|}{ Gender } \\
\hline Female & $27(44.3)$ & $14(41.2)$ & $41(43.2)$ & \multirow{2}{*}{0.002} & \multirow{2}{*}{0.962} \\
\hline Male & $34(55.7)$ & $18(52.9)$ & $52(54.7)$ & & \\
\hline \multicolumn{6}{|l|}{ Age (years) } \\
\hline$\leq 25$ & $46(75.4)$ & 19 (55.9) & $65(68.4)$ & \multirow{2}{*}{2.565} & \multirow{2}{*}{0.109} \\
\hline$>25$ & 15 (24.6) & $13(38.2)$ & $28(29.5)$ & & \\
\hline \multicolumn{6}{|l|}{ Religion } \\
\hline Islam & 41 (67.2) & $30(88.2)$ & 71 (74.7) & \multirow{2}{*}{4.196} & \multirow{2}{*}{0.041} \\
\hline Christianity & $18(29.5)$ & $4(11.8)$ & $22(23.2)$ & & \\
\hline \multicolumn{6}{|l|}{ Tribe } \\
\hline Hausa & $6(9.8)$ & $19(55.9)$ & $25(26.3)$ & \multirow{8}{*}{52.207} & \multirow{8}{*}{$<0.001^{F}$} \\
\hline Kanuri & $14(23.0)$ & $0(0.0)$ & $14(14.7)$ & & \\
\hline Fulani & $3(4.9$ & $8(23.5)$ & 11 (11.6) & & \\
\hline Yoruba & $3(4.9)$ & 7 (20.6) & $10(10.5)$ & & \\
\hline Marghi & $4(6.6)$ & $0(0.0)$ & $4(4.2)$ & & \\
\hline Bura & $4(6.6)$ & $0(0.0)$ & $4(4.2)$ & & \\
\hline Babur & $4(6.6)$ & $0(0.0)$ & $4(4.2)$ & & \\
\hline Others* & $22(36.1)$ & $0(0.0)$ & $23(24.2)$ & & \\
\hline
\end{tabular}

$F=$ Fisher's Exact Test

${ }^{*}$ Kilba, Kibaku, Jaba, Glada, Tera, Nurkum, Kaka, Ngium, Ibibio, Chibok, Kamwe, Bandawa
Participants' responses to the ethical dilemmas and professionalism statements are reported in Table II. More than $79 \%$ of the study population strongly agreed that their actions as future pharmacists could affect how the profession is viewed by patients and other healthcare providers. Additionally, $72.3 \%$ strongly agreed they were respectful of individuals who have backgrounds different from their own. Only $35.1 \%$ of the study participants strongly agreed they knew how to apply the code of ethics in dealing with the ethical dilemma in practice.

When the respondents' views on ethical dilemmas and professionalism were compared between the two Universities studied, it was found that the majority of respondents (59.0\%) from the UM felt strongly that they were aware of the ethical dilemmas that would arise in pharmacy practice. A simple majority of the participants (57.4\%) from the UM were of the strong view that they felt confident in their ability to handle ethical dilemmas that would arise in their future pharmacy practice. Significantly more respondents (51.5\%) from UDU strongly agreed that they knew how to apply the oath of a pharmacist in dealing with ethical dilemmas in practice compared with $26.2 \%$ from the UM $(t(92)=-2.056$; $p=0.043$ ) (Table II).

Table III shows the application of bioethical principles of the respondents. For all the scenarios, participants relied more on the principle of the patient's right to autonomy and non-maleficence as reasons that guide their decisions for dispensing or not dispensing drugs. Respondents' reasons/principles guiding dispensing or not dispensing drugs varied depending on the scenarios. On the use of placebo medication, most respondents (41.8\%) considered the principle of patient autonomy. For the scenario involving sedation of an unruly patient, principles of non-maleficence $(27.5 \%)$ and that of pharmacist's autonomy (24.2\%) were considered more by the participants. For the scenarios pertaining to euthanasia (assisted dying) and expired medications, most of the respondents preferred the principle of non-maleficence (50.5\% and $33.7 \%$ respectively).

Responses of students from both Universities were also compared using Fisher's exact test at $p<0.05$. For scenarios on euthanasia (assisted dying) (60.7\% versus $30 \%$ ) and expired medications (39.3\% versus $22.6 \%$ ), students from the UM relied more on the principle of nonmaleficence. However, students from UDU relied more on the principle of pharmacist's right to autonomy for euthanasia (assisted dying) (36.7\% versus 16.4\%) and expired medications (38.7\% versus $18.0 \%)$ scenarios. Students from both Universities utilised significantly similar bioethical principles when handling placebo and sedation of unruly patient scenarios (Table III). 
Table II: Participants' responses on ethical dilemmas and professionalism statements

\begin{tabular}{|c|c|c|c|c|c|c|c|c|c|c|c|c|c|}
\hline \multirow[t]{2}{*}{ Questionnaire Item } & \multicolumn{2}{|c|}{ Total } & \multicolumn{2}{|c|}{$\begin{array}{l}\text { Strongly } \\
\text { disagree }\end{array}$} & \multicolumn{2}{|c|}{$\begin{array}{l}\text { Somewhat } \\
\text { disagree }\end{array}$} & \multicolumn{2}{|c|}{$\begin{array}{l}\text { Somewhat } \\
\text { agree }\end{array}$} & \multicolumn{2}{|c|}{ Strongly agree } & \multicolumn{2}{|c|}{$\begin{array}{l}\text { Mean score } \\
\text { (SD) }\end{array}$} & \multirow{2}{*}{$p$-value } \\
\hline & $\underset{(\%)}{\operatorname{UM} N}$ & $\begin{array}{l}\text { UDU N } \\
\text { (\%) }\end{array}$ & $\begin{array}{l}\text { UM n } \\
(\%)\end{array}$ & $\begin{array}{l}\text { UDU n } \\
\text { (\%) }\end{array}$ & $\begin{array}{l}\text { UM n } \\
(\%)\end{array}$ & $\begin{array}{l}\text { UDU n } \\
\text { (\%) }\end{array}$ & $\underset{(\%)}{\text { UM } n}$ & $\begin{array}{l}\text { UDU n } \\
\text { (\%) }\end{array}$ & $\underset{(\%)}{\operatorname{UM} n}$ & $\begin{array}{l}\text { UDU n } \\
\text { (\%) }\end{array}$ & UM & UDU & \\
\hline $\begin{array}{l}\text { I am aware of ethical dilemmas } \\
\text { that arise in pharmacy practice. }\end{array}$ & $\begin{array}{c}61 \\
(100)\end{array}$ & $\begin{array}{c}33 \\
(100)\end{array}$ & $\begin{array}{c}3 \\
(4.9)\end{array}$ & $\begin{array}{c}1 \\
(3.0)\end{array}$ & $\begin{array}{c}1 \\
(1.6)\end{array}$ & $\begin{array}{c}1 \\
(3.0)\end{array}$ & $\begin{array}{c}21 \\
(34.4)\end{array}$ & $\begin{array}{c}15 \\
(45.5)\end{array}$ & $\begin{array}{c}36 \\
(59.0)\end{array}$ & $\begin{array}{c}16 \\
(48.5)\end{array}$ & $\begin{array}{c}3.5 \\
(0.8)\end{array}$ & $\begin{array}{c}3.4 \\
(0.7)\end{array}$ & 0.614 \\
\hline $\begin{array}{l}\text { I feel confident in my ability to } \\
\text { handle ethical dilemmas that will } \\
\text { arise in my future pharmacy } \\
\text { practice. }\end{array}$ & $\begin{array}{c}61 \\
(100)\end{array}$ & $\begin{array}{c}33 \\
(100)\end{array}$ & $\begin{array}{c}2 \\
(3.3)\end{array}$ & $\begin{array}{c}1 \\
(3.0)\end{array}$ & $\begin{array}{c}0 \\
(0.0)\end{array}$ & $\begin{array}{c}0 \\
(0.0)\end{array}$ & $\begin{array}{c}24 \\
(39.3)\end{array}$ & $\begin{array}{c}16 \\
(48.5)\end{array}$ & $\begin{array}{c}35 \\
(57.4)\end{array}$ & $\begin{array}{c}16 \\
(48.5)\end{array}$ & $\begin{array}{c}3.5 \\
(0.7)\end{array}$ & $\begin{array}{c}3.4 \\
(0.7)\end{array}$ & 0.563 \\
\hline $\begin{array}{l}\text { I know how to apply the oath of a } \\
\text { Pharmacist to resolve dilemmas } \\
\text { in pharmacy practice. }\end{array}$ & $\begin{array}{c}61 \\
(100)\end{array}$ & $\begin{array}{c}33 \\
(100)\end{array}$ & $\begin{array}{c}5 \\
(8.2)\end{array}$ & $\begin{array}{c}1 \\
(3.0)\end{array}$ & $\begin{array}{c}6 \\
(9.8)\end{array}$ & $\begin{array}{c}3 \\
(9.1)\end{array}$ & $\begin{array}{c}34 \\
(55.7)\end{array}$ & $\begin{array}{c}12 \\
(36.4)\end{array}$ & $\begin{array}{c}16 \\
(26.2)\end{array}$ & $\begin{array}{c}17 \\
(51.5)\end{array}$ & $\begin{array}{c}3.0 \\
(0.8)\end{array}$ & $\begin{array}{c}3.4 \\
(0.8)\end{array}$ & $0.043^{*}$ \\
\hline $\begin{array}{l}\text { I know how to apply the } \\
\text { Pharmacists Code of Ethics to } \\
\text { resolve dilemmas in pharmacy } \\
\text { practice. }\end{array}$ & $\begin{array}{c}61 \\
(100)\end{array}$ & $\begin{array}{c}32 \\
(100)\end{array}$ & $\begin{array}{c}3 \\
(4.9)\end{array}$ & $\begin{array}{c}0 \\
(0.0)\end{array}$ & $\begin{array}{c}5 \\
(8.2)\end{array}$ & $\begin{array}{c}4 \\
(12.5)\end{array}$ & $\begin{array}{c}30 \\
(49.2)\end{array}$ & $\begin{array}{c}15 \\
(46.9)\end{array}$ & $\begin{array}{c}23 \\
(37.7)\end{array}$ & $\begin{array}{c}13 \\
(40.6)\end{array}$ & $\begin{array}{c}3.2 \\
(0.8)\end{array}$ & $\begin{array}{c}3.3 \\
(0.7)\end{array}$ & 0.610 \\
\hline $\begin{array}{l}\text { My actions as a future pharmacist } \\
\text { impact how patients view the } \\
\text { profession of pharmacy. }\end{array}$ & $\begin{array}{c}61 \\
(100)\end{array}$ & $\begin{array}{c}32 \\
(100)\end{array}$ & $\begin{array}{c}2 \\
(3.3)\end{array}$ & $\begin{array}{c}1 \\
(3.1)\end{array}$ & $\begin{array}{c}1 \\
(1.6)\end{array}$ & $\begin{array}{c}0 \\
(0.0)\end{array}$ & $\begin{array}{c}12 \\
(19.7)\end{array}$ & $\begin{array}{c}4 \\
(12.5)\end{array}$ & $\begin{array}{c}47 \\
(77.0)\end{array}$ & $\begin{array}{c}27 \\
(84.4)\end{array}$ & $\begin{array}{c}3.7 \\
(0.6)\end{array}$ & $\begin{array}{c}3.8 \\
(0.6)\end{array}$ & 0.580 \\
\hline $\begin{array}{l}\text { My actions as a future pharmacist } \\
\text { impact how the profession of } \\
\text { pharmacy is viewed by other } \\
\text { health care providers. }\end{array}$ & $\begin{array}{c}61 \\
(100)\end{array}$ & $\begin{array}{c}33 \\
(100)\end{array}$ & $\begin{array}{c}2 \\
(3.3)\end{array}$ & $\begin{array}{c}1 \\
(3.0)\end{array}$ & $\begin{array}{c}1 \\
(1.6)\end{array}$ & $\begin{array}{c}2 \\
(6.1)\end{array}$ & $\begin{array}{c}8 \\
(13.1)\end{array}$ & $\begin{array}{c}4 \\
(12.1)\end{array}$ & $\begin{array}{c}50 \\
(82.0)\end{array}$ & $\begin{array}{c}26 \\
(78.8)\end{array}$ & $\begin{array}{c}3.7 \\
(0.7)\end{array}$ & $\begin{array}{c}3.7 \\
(0.7)\end{array}$ & 0.632 \\
\hline $\begin{array}{l}\text { I am diplomatic when expressing } \\
\text { ideas and opinions. }\end{array}$ & $\begin{array}{c}60 \\
(100)\end{array}$ & $\begin{array}{c}33 \\
(100)\end{array}$ & $\begin{array}{c}3 \\
(5.0)\end{array}$ & $\begin{array}{c}0 \\
(0.0)\end{array}$ & $\begin{array}{c}4 \\
(6.7)\end{array}$ & $\begin{array}{c}2 \\
(6.1)\end{array}$ & $\begin{array}{c}25 \\
(41.7)\end{array}$ & $\begin{array}{c}16 \\
(48.5)\end{array}$ & $\begin{array}{c}28 \\
(46.7)\end{array}$ & $\begin{array}{c}15 \\
(45.5)\end{array}$ & $\begin{array}{c}3.3 \\
(0.8)\end{array}$ & $\begin{array}{c}3.4 \\
(0.6)\end{array}$ & 0.562 \\
\hline $\begin{array}{l}\text { I am respectful of individuals who } \\
\text { have different backgrounds than } \\
\text { mine. }\end{array}$ & $\begin{array}{c}61 \\
(100)\end{array}$ & $\begin{array}{c}33 \\
(100)\end{array}$ & $\begin{array}{c}1 \\
(1.6)\end{array}$ & $\begin{array}{c}1 \\
(3.0)\end{array}$ & $\begin{array}{c}3 \\
(4.9)\end{array}$ & $\begin{array}{c}0 \\
(0.0)\end{array}$ & $\begin{array}{c}11 \\
(18.0)\end{array}$ & $\begin{array}{c}10 \\
(30.3)\end{array}$ & $\begin{array}{c}46 \\
(75.4)\end{array}$ & $\begin{array}{c}22 \\
(66.7)\end{array}$ & $\begin{array}{l}3.7 \\
(0.7)\end{array}$ & $\begin{array}{c}3.6 \\
(0.7)\end{array}$ & 0.641 \\
\hline
\end{tabular}

UM = University of Maiduguri; UDU = Usmanu Danfodiyo University

${ }^{*} t$-test $p<0.05$

Table III: Participants' perceived application of bioethical principles

\begin{tabular}{|c|c|c|c|c|c|c|c|c|c|c|c|c|}
\hline \multirow[t]{3}{*}{ Bioethical principle } & \multirow{2}{*}{\multicolumn{3}{|c|}{$\begin{array}{c}\text { Placebo medication } \\
\text { Pharmacy School }\end{array}$}} & \multirow{2}{*}{\multicolumn{3}{|c|}{$\begin{array}{c}\text { Sedation } \\
\text { Pharmacy School } \\
\end{array}$}} & \multirow{2}{*}{\multicolumn{3}{|c|}{$\begin{array}{c}\text { Euthanasia } \\
\text { Pharmacy School } \\
\end{array}$}} & \multirow{2}{*}{\multicolumn{3}{|c|}{$\begin{array}{c}\text { Expired medication } \\
\text { Pharmacy School } \\
\end{array}$}} \\
\hline & & & & & & & & & & & & \\
\hline & $\begin{array}{l}\text { Total } \\
\mathbf{N}(\%)\end{array}$ & $\begin{array}{l}\text { UM } \\
\mathrm{n}(\%)\end{array}$ & $\begin{array}{l}\text { UDU } \\
\text { n (\%) }\end{array}$ & $\begin{array}{l}\text { Total } \\
\mathbf{N}(\%)\end{array}$ & $\begin{array}{l}\text { UM } \\
\mathrm{n}(\%)\end{array}$ & $\begin{array}{l}\text { UDU } \\
\text { n (\%) }\end{array}$ & $\begin{array}{l}\text { Total } \\
\text { N (\%) }\end{array}$ & $\begin{array}{l}\text { UM } \\
\mathrm{n}(\%)\end{array}$ & $\begin{array}{l}\text { UDU } \\
\text { n (\%) }\end{array}$ & $\begin{array}{l}\text { Total } \\
\mathbf{N}(\%)\end{array}$ & $\begin{array}{l}\text { UM } \\
\mathrm{n}(\%)\end{array}$ & $\begin{array}{l}\text { UDU } \\
\mathrm{n}(\%)\end{array}$ \\
\hline Patient autonomy & $38(41.8)$ & $23(37.7)$ & $15(50.0)$ & $7(7.7)$ & $2(3.3)$ & $5(16.7)$ & $13(14.3)$ & $5(8.2)$ & $8(26.7)$ & $9(9.8)$ & $0(0.0)$ & $9(29.0)$ \\
\hline Pharmacist autonomy & $8(8.8)$ & $4(6.6)$ & $4(13.3)$ & $22(24.2)$ & $14(23.0)$ & $8(26.7)$ & $21(23.1)$ & $10(16.4)$ & $11^{*}(36.7)$ & $23(25.0)$ & $11(18.0)$ & $12^{*}(38.7)$ \\
\hline Non-maleficence & $17(18.7)$ & $9(14.8)$ & $8(26.7)$ & $25(27.5)$ & $14(23.0)$ & $11(36.7)$ & $46(50.5)$ & $37^{*}(60.7)$ & $9(30.0)$ & $31(33.7)$ & $24^{*}(39.3)$ & $7(22.6)$ \\
\hline Benevolence & $4(4.4)$ & $4(6.6)$ & $0(0.0)$ & $11(12.1)$ & $9(14.8)$ & $2(6.7)$ & $1(1.1)$ & $1(1.6)$ & $0(0.0)$ & $1(1.1)$ & $1(1.6)$ & $0(0.0)$ \\
\hline Veracity & $13(14.3)$ & $12(19.7)$ & $1(3.3)$ & $11(12.1)$ & $10(16.4)$ & $1(3.3)$ & $6(6.6)$ & $6(9.8)$ & $0(0.0)$ & $27(29.3)$ & $24^{*}(39.3)$ & $3(9.7)$ \\
\hline Justice & $2(2.2)$ & $2(3.3)$ & $0(0.0)$ & $2(2.2)$ & $2(3.3)$ & $0(0.0)$ & $1(1.1)$ & $1(1.6)$ & $0(0.0)$ & $1(1.1)$ & $1(1.6)$ & $0(0.0)$ \\
\hline Fidelity to Doctor & $7(7.7)$ & $6(9.8)$ & $1(3.3)$ & $11(12.1)$ & $9(14.8)$ & $2(6.7)$ & $1(1.1)$ & $1(1.6)$ & $0(0.0)$ & $0(0.0)$ & $0(0.0)$ & $0(0.0)$ \\
\hline $\begin{array}{l}\text { Patient-Pharmacist } \\
\text { relationship }\end{array}$ & $2(2.2)$ & $1(1.6)$ & $1(3.3)$ & $2(2.2)$ & $1(1.6)$ & $1(3.3)$ & $2(2.2)$ & $0(0.0)$ & $2(6.7)$ & $0(0.0)$ & $0(0.0)$ & $0(0.0)$ \\
\hline Total & 91 (100) & $61(100)$ & $30(100)$ & $91(100)$ & $61(100)$ & 30 (100) & 91 (100) & $61(100)$ & $30(100)$ & $92(100)$ & 61 (100) & 31 (100) \\
\hline
\end{tabular}

Bold font represents bioethical principle with highest frequency per sample group per scenario

*Fisher's Exact Test $p<0.05$ 
Table IV: Participants' perceived application of bioethical principles categorised by gender and religion

\begin{tabular}{|c|c|c|c|c|c|c|c|c|}
\hline \multirow[t]{2}{*}{ Bioethical principle } & \multicolumn{2}{|c|}{ Placebo medication } & \multicolumn{2}{|c|}{ Sedation } & \multicolumn{2}{|c|}{ Euthanasia } & \multicolumn{2}{|c|}{ Expired medication } \\
\hline & $\begin{array}{c}\text { Female } \\
\mathrm{n}(\%)\end{array}$ & $\begin{array}{l}\text { Male } \\
\mathrm{n}(\%)\end{array}$ & $\begin{array}{c}\text { Female } \\
\text { n (\%) }\end{array}$ & $\begin{array}{l}\text { Male } \\
\mathrm{n}(\%)\end{array}$ & $\begin{array}{l}\text { Female } \\
\text { n (\%) }\end{array}$ & $\begin{array}{l}\text { Male } \\
\mathrm{n}(\%)\end{array}$ & $\begin{array}{l}\text { Female } \\
\text { n (\%) }\end{array}$ & $\begin{array}{l}\text { Male } \\
\mathrm{n}(\%)\end{array}$ \\
\hline Patient autonomy & $18(46.2)$ & $18(36.0)$ & $4(10.0)$ & $2(4.0)$ & $5(12.8)$ & $7(13.7)$ & $3(7.5)$ & $5(9.8)$ \\
\hline Pharmacist autonomy & $4(10.3)$ & $4(8.0)$ & $6(15.0)$ & $16(32.0)$ & $6(15.4)$ & $15(29.4)$ & $9(22.5)$ & $14(27.5)$ \\
\hline Non-maleficence & 7 (17.9) & $10(20.0)$ & $14(35.0)$ & $11(22.0)$ & $25(64.1)$ & $21(41.2)$ & $15(37.5)$ & $16(31.4)$ \\
\hline Benevolence & $1(2.6)$ & $3(6.00$ & $5(12.5)$ & $6(12.0)$ & $0(0.0)$ & $1(2.0)$ & $0(0.0)$ & $1(2.0)$ \\
\hline Veracity & $6(15.4)$ & $7(14.0)$ & $5(12.5)$ & $6(12.0)$ & $2(5.1)$ & $4(7.8)$ & $12(30.0)$ & $15(29.4)$ \\
\hline Justice & $1(2.6)$ & $1(2.0)$ & $0(0.0)$ & $2(4.0)$ & $0(0.0)$ & $1(2.0)$ & $1(2.5)$ & $0(0.0)$ \\
\hline Fidelity to Doctor & $2(5.1)$ & $5(10.0)$ & $5(12.5)$ & $6(12.0)$ & $1(2.6)$ & $0(0.0)$ & $0(0.0)$ & $0(0.0)$ \\
\hline $\begin{array}{l}\text { Patient-Pharmacist } \\
\text { relationship }\end{array}$ & $0(0.0)$ & $2(4.0)$ & $1(2.5)$ & $1(2.0)$ & $0(0.0)$ & $2(3.9)$ & $0(0.0)$ & $0(0.0)$ \\
\hline Total & $39(100)$ & $50(100)$ & $40(100)$ & $50(100)$ & 39 (100) & $51(100)$ & $40(100)$ & $51(100)$ \\
\hline Bioethical principle & $\begin{array}{l}\text { Islam } \\
\text { n (\%) }\end{array}$ & $\begin{array}{l}\text { Christianity } \\
\text { n (\%) }\end{array}$ & $\begin{array}{l}\text { Islam } \\
\text { n (\%) }\end{array}$ & $\begin{array}{l}\text { Christianity } \\
\text { n (\%) }\end{array}$ & $\begin{array}{l}\text { Islam } \\
\text { n (\%) }\end{array}$ & $\begin{array}{l}\text { Christianity } \\
\text { n (\%) }\end{array}$ & $\begin{array}{l}\text { Islam } \\
\text { n (\%) }\end{array}$ & $\begin{array}{l}\text { Christianity } \\
\text { n (\%) }\end{array}$ \\
\hline Patient autonomy & $29(42.6)$ & $8(38.1)$ & $6(9.0)$ & $1(4.5)$ & $10(14.9)$ & $3(13.6)$ & $8(11.8)$ & $1(4.5)$ \\
\hline Pharmacist autonomy & $8(11.8)$ & $0(0.0)$ & $15(22.4)$ & $7(31.8)$ & $17(25.4)$ & $4(18.2)$ & $17(25.0)$ & $6(27.3$ \\
\hline Non-maleficence & 13 (19.1) & $3(14.3)$ & 20 (29.9) & $5(22.7)$ & $33(49.3)$ & $12(54.5)$ & $22(32.4)$ & $9(40.9)$ \\
\hline Benevolence & $4(5.9)$ & $0(0.0)$ & 10 (14.9) & $1(4.5)$ & $1(1.5)$ & $0(0.0)$ & $1(1.5)$ & $0(0.0)$ \\
\hline Veracity & 10 (14.7) & $3(14.3)$ & 8 (11.9) & $2(9.1)$ & $2(3.0)$ & $3(13.6)$ & $19(27.9)$ & $6(27.3)$ \\
\hline Justice & $1(1.5)$ & $1(4.8)$ & $1(1.5)$ & $1(4.5)$ & $1(1.5)$ & $0(0.0)$ & $1(1.5)$ & $0(0.0)$ \\
\hline Fidelity to Doctor & $2(2.9)$ & $5(23.8)$ & $5(7.5)$ & $5(22.7)$ & $1(1.5)$ & $0(0.0)$ & $0(0.0)$ & $0(0.0)$ \\
\hline $\begin{array}{l}\text { Patient-Pharmacist } \\
\text { relationship }\end{array}$ & $1(1.5)$ & $1(4.8)$ & $2(3.0)$ & $0(0.0)$ & $2(3.0)$ & $0(0.0)$ & $0(0.0)$ & $0(0.0)$ \\
\hline Total & $68(100)$ & 21 (100) & $67(100)$ & $22(100)$ & 67 (100) & $22(100)$ & 68 (100) & $22(100)$ \\
\hline
\end{tabular}

Bold font represents bioethical principle with highest frequency per sample group per scenario

Furthermore, the effect of age ( $\leq 25$ and $>25$ years), gender (male and female) and religion (Muslims and Christians) on dispensing medication based on different practice scenarios was also evaluated. The difference in age group had no effect on the perceived application of bioethical principles. The perceived bioethical principle of the respondents differed only for the scenarios pertaining to sedation as shown in Table IV. Female (35.0\%) and Muslim (29.9\%) respondents preferred the principle of non-maleficence. On the other hand, male (32.0\%) and Christian (31.8\%) respondents preferred the principle of pharmacist's autonomy.

\section{Discussion}

In terms of professionalism, the understanding of the students that their actions as future pharmacists impact on how the patients and other healthcare providers view the pharmacy profession was apparent. In contrast, a low proportion of these students strongly agreed that they knew how to apply the pharmacist's oath and code of ethics to resolve the ethical dilemmas, and being diplomatic when expressing opinions. An earlier study from a United States' (US) pharmacy school reported lower values than this study regarding the application of 
professional oath and codes of ethics to resolving the ethical dilemma in the pre-intervention phase with dramatic improvement after the institution of an intervention (early exposure to professionalism and ethical dilemmas) (Smith \& Dinkins, 2015). These findings imply that the didactic mode of delivery of pharmacy ethics courses and lack of early exposure of students to professionalism and ethical dilemmas is not just a problem for pharmacy education in Nigeria, but for other countries as well. This highlights the need for the inclusion of practical components such as small group discussions and case debates among others that would allow the application of the professional oath and ethics to various culturally adapted ethical dilemmas encountered in contemporary pharmacy practice in the pharmacy ethics syllabi globally. Since the objective of the ethics education is to provide practice skills, this must be kept in mind when the content of ethics syllabi is determined, and attention to bioethical issues are required if pharmacy students are to provide high quality patient-centred care after graduation (Ibrahim \& Sharif, 2018).

The mean knowledge score of the perceived ability to apply the pharmacist's oath was significantly lower among the students at the UM compared to their counterparts at the UDU. The difference in ethics syllabi and mode of delivery of this course could account for this observation. Nonetheless, some previous studies among community pharmacists (Hibbert et al., 2000; Chaar et al., 2005; Cooper, 2007) appeared to cast doubt on the relevance of a pharmacy code alone in resolving ethical dilemmas. This underscores the need to teach students to always supplement the professional code with ethical decision-making models and bioethical textbooks due to the imperfection of this code.

With respect to the utilisation of the bioethical principles to ethical dilemmas by the participants, overall, this study showed that these students utilised the patient's right to autonomy for the placebo scenario, and non-maleficence for both sedation, and the expired medication scenarios consistent with the findings of a previous study conducted in the US among Pharm.D. students (Schlesselman, 2014). This highlights that these students considered the patient's right to make decisions about his/her health without the interference of healthcare providers and their safety as paramount. Contemporary ethics in pharmacy practice emphasise that the dignity and welfare of patients must be paramount. However, it is only a competent patient that can exercise the right to autonomy to its fullest (Kihlbom, 2008), this right can only be overridden if there is substantial threat to other people (Resnik, 2005). Contrary to the patient's right to autonomy and non-maleficence utilised by students for placebo and sedation scenarios, respectively, the pharmacist's right to autonomy and pharmacist-physician relationship were at stake here. Additionally, in this study, these students relied on non-maleficence for euthanasia (assisted dying) scenario, which is inconsistent with a previous study done in the US pharmacy school which reported that patient autonomy was more commonly used (Schlesselman, 2014). The plausible reasons for this could be variations in programmes, teaching methods, educational and legal systems, culture, and geographic location. In agreement with the authors' study participants, in Nigeria human life is sacrosanct. Therefore, physicians and pharmacists are not allowed to prescribe and dispense lethal medicines to patients who may request them. So, a patient who is fully informed of all available care options, has a clear and settled intention to die, and is competent to voluntarily decide without compulsion does not need to involve any healthcare practitioner (Hurley, 2020).

Participants' responses to sedation and expired medication scenarios significantly differed among pharmacy schools. For the sedation scenario, a significant proportion of the students at UDU were more decisive than their peers at the UM that the pharmacist should dispense the drug, justifying their actions more on the pharmacist's autonomy. This suggests that students at UDU added legal under-tone to it because the pharmacist is mandated by law to dispense medicines to the right person. On the other hand, a significant proportion of the students at the UM were more decisive than their counterparts at the UDU that the pharmacist should not accept expired medications for a medical mission trip, justifying their actions on non-maleficence. This result could suggest that these students felt they had no right to overrule the drug regulatory agency's mandated expiration dates of medications. Their position may indicate that pharmacists accepting and sending the expired medications to people that were already suffering and helpless is not acting in the best interests of the people. To corroborate this choice, the World Health Organisation (WHO) vehemently frowns on the donation of expired medications, even to affected nations during an emergency or crisis (WHO, 1999). The observed difference among the students from both pharmacy schools may in part be due to variations in specific professional ethics training with reference to pharmacy practice bioethical dilemmas. Therefore, these findings underscore the need to harmonise the ethics syllabi for pharmacy law and ethics courses of various pharmacy schools in Nigeria. However, the finding of this study is inconsistent with that 
of a previous study done in the US pharmacy school that reported pharmacist's right to autonomy for expired medication scenario (Schlesselman, 2014).

Based on gender and religious group, the sedation scenario elicited different responses which were not statistically significant but may be of practical significance. In this case scenario, male and Christian pharmacy students were more decisive than their female and Muslim peers that the pharmacist should dispense the medication, justifying their actions more on pharmacist's right to autonomy. These findings demonstrate that male and Christian pharmacy students were of the view, that the pharmacist has a right to override the patient's autonomy when a patient is incompetent or the pharmacist is acting for their best interest as against dispense and do no harm position of their female and Muslim counterparts. This is in line with the International Pharmacy Federation (FIP) report that pharmacist's ethics and professional autonomy is imperative for keeping pharmacy aligned with the community demands (FIP, 2013).

In summary, how Nigerian pharmacy students perceived their learning of professionalism and bioethics from the pharmacy law and ethics course has not been previously assessed. Therefore, the implications of this study's findings for professionalism and ethics teaching in the pharmacy schools taking part on the study are that the current pharmacy law and ethics courses may not be adequate to impact bioethical reasoning knowledge and skills in their students. This reinforces the need for pharmacy educators to review and improve the contents of this course with bioethics. Alternatively, separate professionalism and bioethics enriched courses could be designed to equip the students with the requisite professionalism and bioethics knowledge and skills necessary for contemporary pharmacy practice. In addition, mixed teaching methods such as didactic mode of delivery, case-based learning, debates, group discussions and presentations among others should be used to holistically prepare these students for improved patient-centred practice upon graduation.

\section{Limitations}

The main limitation of this study is the small sample size; therefore, the findings cannot be generalised to other pharmacy schools in Nigeria. Some students who may have not been exposed to the moral dilemma expressed in the study cases may not adequately understand the moral implications of the cases. Other limitations are the possibility of response and social desirability bias due to the tendency of some students to give self-positive perceptions which may not reflect the truth. Lastly, paucity of data with respect to pharmacy students' professionalism and application of bioethics principles could not allow for an exhaustive discussion of the study key findings.

\section{Conclusion}

Pharmacy students at UM relied more on the principles of non-maleficence, while those at UDU considered pharmacist's autonomy more frequently. Females and males, and Islamic and Christian students relied more on the principles of non-maleficence to support how they would likely handle each ethical dilemma.

The study has shown that pharmacy students about to enter a healthcare profession did not have high perceived ethical reasoning. The results of this study provide further evidence for the need for ethical instruction in the profession of pharmacy. Therefore, redesigning of the pharmacy law and ethics course and teaching method is highly critical for the students in providing effective patient-centred pharmacy practice and to make sound clinical decisions at all times in practice upon graduation.

Finally, this work adds to the knowledge concerning this area of pharmacy education, although further studies are warranted to assess the moral development of final-year pharmacy students in these pharmacy schools and deeper exploration of their ethical decision making when presented with various contemporary dilemmas encountered in pharmacy practice.

\section{Acknowledgments}

The authors wish to express their profound gratitude to the students who participated in this study.

\section{Conflicts of interest}

The authors declare that they have no conflicts of interest. 


\section{References}

Abdu-aguye, S.N., Yusuf, H., Agbale, E.O., Auwal, F.I., \& Ma'aji, H.U. (2019). Connections between classroom theory \& real world practice: Exploring the perspectives of undergraduate students at a Nigerian faculty of pharmacy. Pharmacy Education, 19(1), 185-189

Beauchamp, T., \& Childress, J. (2001). Principles of biomedical ethics. New York: Oxford University Press

Benson, A., Cribb, A., \& Barber, N. (2009). Understanding pharmacists' values: a qualitative study of ideals and dilemmas in UK pharmacy practice. Social Science \& Medicine, 68(12), 2223-2230. https://doi.org/10.1016/i.socscimed.2009.03.012

Buchanan, R. (2017). Cases of unethical behavior in pharmacy (online). Available at: https://bizfluent.com/facts-5828012-sellsbreck-shampoo-.html

Chaar, B., Brien, J. \& Krass, I. (2005). Professional ethics in pharmacy: the Australian experience. International Journal of Pharmacy Practice, 13(3), 195-204. https://doi.org/10.1211/ijpp. $\underline{13.3 .0005}$

Cooper, R.J., Bissell, P., \& Wingfield, J. (2007). A new prescription for empirical ethics research in pharmacy: a critical review of the literature. Journal of Medical Ethics, 33(2), 82-86. https:// doi.org/10.1136/ime.2005.015297

Cooper, R.J. (2007). Ethical problems and their resolution amongst UK community pharmacists: a qualitative study. PhD thesis, University of Nottingham (online). Available at: http:// eprints.nottingham.ac.uk/10265/1/6gpdf.pdf

Cooper, R.J., Bissell, P., \& Wingfield, J. (2008). Ethical decisionmaking, passivity and pharmacy. Journal of Medical Ethics, 34(6), 441-445. https://doi.org/10.1136/ime.2007.022624

Donaldson, T.M., Fistein, E., \& Dunn, M. (2010). Case-based seminars in medical ethics education: how medical students define and discuss moral problems. Journal of Medical Ethics, 36(12), 816-820. https://doi.org/10.1136/jme.2010.036574

Erah, P. (2011). The PharmD Programme; Prospects and challenges in Nigeria. Nigerian Journal of Pharmaceutical Research, 9(1), 30-48

Fakeye, T., Adisa, R., \& Erhun, W. (2017). Developing a model for teaching and learning clinical pharmacy component $s$ of the pharmacy curriculum in Nigeria. Pharmacy Education, 17(1), 60-66

FIP [International Pharmaceutical Federation]. (2013). Reference Document - Pharmacist Ethics and Professional Autonomy: Imperatives for Keeping Pharmacy Aligned with the Public Interest. International Pharmaceutical Federation (online). Available at: https://www.fip.org/file/1368

Frunză, M. (2011). Case studies in teaching euthanasia: ethical and pedagogical aspects. Revista Romana De Bioetica, 9, 108-113

Gallagher, C.T. (2011). Assessment of levels of moral reasoning in pharmacy students at different stages of the undergraduate curriculum. International Journal of Pharmacy Practice, 19(5), 374-380. https://doi.org/10.1111/i.2042-7174.2011.00121.x
Hanna, L., Gillen, J., \& Hall, M. (2017). An investigation of final year pharmacy students' moral reasoning ability, and their views on professionalism and fitness to practice panel determinations: A questionnaire study. Current in Pharmacy Teaching and Learning, 9(4), 652-659. https://doi.org/10.1016/j.cptl.2017.03.016

Henning, M.A., Malpas, P., Ram, S., Rajput, V., Krstić, V., Boyd, M., \& Hawken, S.J. (2016). Students' responses to scenarios depicting ethical dilemmas: a study of pharmacy and medical students in New Zealand. Journal of Medical Ethics, 42(7), 466-473. https://doi.org/10.1136/medethics-2015-103253

Hibbert, D., Rees, J.A., \& Smith, I. (2000). Ethical awareness of community pharmacists. International Journal of Pharmacy Practice, 8(2), 82-87. https://doi.org/10.1111/i.2042-7174.2000. tb00990.x

Hurley, R. (2020). Assisted dying: your vote counts. British Medical Journal, 368(m646). https://doi.org/10.1136/bmj.m646

Ibrahim, R.M., \& Sharif, S.I. (2018). Teaching professionalism and ethical reasoning to pharmacy students: How important it is? Global Drugs Therapy, 3(5), 1-1. https://doi.org/10.15761/GDT. $\underline{1000162}$

Ikhile, I.J., \& Chijioke-Nwauche, I.N. (2016). Pharmacy education in Nigeria: The progression. World Journal of Pharmaceutical Research, 5(7), 258-272

Johnson, J.A., \& Bootman, J.L. (1995). Drug-related morbidity and mortality: A cost-of-illness model. Archives of Internal Medicine, 155(18), 1949-1956. https://doi.org/10.1001/archinte. $\underline{1995.00430180043006}$

Johnson, J.A., \& Bootman, J.L. (1997). Drug-related morbidity and mortality and the economic impact of pharmaceutical care. American Journal of Health-System Pharmacy, 54(5), 554-558. https://doi.org/10.1093/ajhp/54.5.554

Kalvemark, S., Hoglund, A.T., Hansson, M.G., Westerholm, P., \& Arnetz, B. (2004). Living with conflicts-ethical dilemmas and moral distress in the health care system. Social Science \& Medicine, 58(6), 1075-1084. https://doi.org/10.1016/S0277$\underline{9536(03) 00279-X}$

Kangasniemi, M., Pakkanen, P., \& Korhonen, A. (2015). Professional ethics in nursing: An integrative review. Journal of Advance Nursing, 7(8), 1-14. https://doi.org/10.1111/jan.12619

Kihlbom, U. (2008). Autonomy and negatively informed consent. Journal of Medical Ethics, 34, 146-149. https://doi.org/10.1136/ ime.2007.020503

Kruijtbosch, M., Göttgens-Jansen, W., Floor-Schreudering, A., Leeuwen, E. \& Bouvy, M.L. (2018). Moral dilemmas of community pharmacists: a narrative study. International Journal of Clinical Pharmacy, 40(1), 74-83. https://doi.org/10.1007/ s11096-017-0561-0

Latif, D.A. (2001). The relationship between pharmacists' tenure in community setting and moral reasoning. Journal of Business Ethics, 31(2), 131-141. https://doi.org/10.1023/A:1010771103427

Latif, D.A. (2003). Moral reasoning and its implications for pharmacy education. Pharmacy Education, 3(1), 1-6 
Latif, D.A. (2004). An assessment of the ethical reasoning of United States pharmacy students: a national study. American Journal of Pharmaceutical Education, 68(2), Art. 30. https:// doi.org/10.5688/aj680103

Lindsey, W.T., \& Olin, B.R. (2014). Student responses to legal and ethical scenarios in drug information. Journal of Pharmaceutical Care \& Health Systems, S1-006. https://doi.org/10.4172/2376-0419.S1-006

McCormick, T.R. (2018). Principle of Bioethics (online). Available at: https://depts.washington.edu/bhdept/ethics-medicine/ bioethics-topics/articles/principles-bioethics

Ogaji, J.I., \& Ojabo, C.E. (2014). Pharmacy education in Nigeria: The journey so far. Archives of Pharmacy Practice, 5(2), 47-60. https://doi.org/10.4103/2045-080X.132644

Okoye, O., Nwachukwu, D., \& Maduka-Okafor, F.C. (2017). Must we remain blind to undergraduate medical ethics education in Africa? A cross-sectional study of Nigerian medical students. BMC Medical Ethics, 18(1), 73. https://doi.org/10.1186/ $\underline{\text { s12910-017-0229-2 }}$

Resnik, D.B. (2005). The patient's duty to adhere to prescribed treatment: An ethical analysis. Journal of Medicine and Physiology, 30, 167-188. https://doi.org/10.1080/036053105909 26849

Schlesselman, L.S. (2014). A cross-sectional study of applied bioethical reasoning in pharmacy students and preceptors. Pharmacy Practice, 12(2), 401-410. https://doi.org/10.4321/ $\underline{\text { S1886- } 36552014000200007}$

Sharif, P.S., Javadi, M., \& Asghari, F. (2011). Pharmacy ethics: evaluation pharmacists' ethical attitude. Journal of Medical Ethics and History of Medicine, 4(5)

Slomka, J., Quill, B., desVignes-Kendrick, M., \& Lloyd, L.E. (2008). Professionalism and Ethics in the Public Health Curriculum. Public Health Reports, Sup2(123), 27-35. https://doi.org/ $\underline{10.1177 / 003335490812305205}$

Smith, M.G., \& Dinkins, M.M. (2015). Instructional design and assessment: early introduction to professional and ethical dilemmas in a pharmaceutical care laboratory course. American Journal of Pharmaceutical Education, 79(10), Art. 156. http:// doi.org/10.5688/ajpe7910156

Tekiner, H. (2017). Why Teach Pharmacy Ethics Through Literary Fiction? World Journal of Pharmaceutical Sciences, 5(6), 203-206

University of Greenwich. (2015). Introduction to Pharmacy Professional Skills, Law and Ethics (online). Available at: https:// www.gre.ac.uk/foundation-degrees//content/

Vivian, J.C. (2010). Rogue Pharmacists. US Pharm, 35(10), 120-123

Warren, T.J. (2011). The 4 basic ethical principles that apply to forensic activities are respect for autonomy, beneficence, nonmaleficence, and justice. Journal of Chiropractic Medicine, 10(3), 225-226. https://doi.org/10.1016/i.jcm.2011.08.004

WHO. (1999). Guidelines for Drug Donations. Available at: https://www.who.int/hac/techguidance/en/

Wingfield, J., Bissell, P., \& Anderson, C. (2004). The scope of pharmacy ethics: an evaluation of the international research literature, 1990-2002. Social Science \& Medicine, 58(12), 2383-2396. https://doi.org/10.1016/j.socscimed.2003.09.003

\section{Appendix A}

Professionalism and Ethical Dilemmas Questionnaire for Student Pharmacists

Professionalism and Ethical Dilemmas Questionnaire for Student Pharmacists

Please fill in the spaces provided and tick [V] the option that appears most appropriate

Section 1: Socio-demographics:

Name of University:

What is your gender? Male [ ] Female [ ]

What is your age? $18-25$ years [ ] 26-30 years [ ] 31-35 years [ ] $36-40$ years [ ] $41-45$ years [ ] over 45 years [ ]

What is your tribe?

What is your religion?

For the following items, tick [ $\mathrm{V}$ ] whether you strongly disagree, somewhat disagree, somewhat agree, or strongly agree with the statement.

\begin{tabular}{|l|l|l|l|l||}
\hline Questionnaire Item & $\begin{array}{c}\text { Strongly } \\
\text { Disagree }\end{array}$ & $\begin{array}{c}\text { Somewhat } \\
\text { Disagree }\end{array}$ & $\begin{array}{c}\text { Somewhat } \\
\text { Agree }\end{array}$ & $\begin{array}{c}\text { Strongly } \\
\text { Agree }\end{array}$ \\
\hline $\begin{array}{l}\text { I am aware of ethical dilemmas } \\
\text { that arise in pharmacy practice }\end{array}$ & & & & \\
\hline $\begin{array}{l}\text { I feel confident in my ability to } \\
\text { handle ethical dilemmas that will } \\
\text { arise in my future pharmacy } \\
\text { practice }\end{array}$ & & & & \\
\hline $\begin{array}{l}\text { I know how to apply the Oath of a } \\
\text { Pharmacist to resolve dilemmas in } \\
\text { pharmacy practice }\end{array}$ & & & & \\
\hline $\begin{array}{l}\text { I know how to apply the Code of } \\
\text { Ethics for Pharmacists to resolve } \\
\text { dilemmas in pharmacy practice }\end{array}$ & & & & \\
\hline $\begin{array}{l}\text { My actions as a future pharmacist } \\
\text { impact how the profession of } \\
\text { pharmacy is viewed by patients }\end{array}$ & & & & \\
\hline $\begin{array}{l}\text { My actions as a future pharmacist } \\
\text { impact how the profession of } \\
\text { pharmacy is viewed by other } \\
\text { health care providers }\end{array}$ & & & & \\
\hline $\begin{array}{l}\text { I am diplomatic when expressing } \\
\text { ideas and opinions }\end{array}$ & & & & \\
\hline $\begin{array}{l}\text { I am respectful of individuals who } \\
\text { have different backgrounds than } \\
\text { mine }\end{array}$ & & & & \\
\hline & & & \\
\hline
\end{tabular}

mine

\section{Section 2: Pharmacy Ethical Dilemmas Survey (PEDS)}

In this section of the survey you will be asked how you would handle pharmacy-specific dilemmas and why you would handle it in this manner. After reading the scenario, mark whether you would or would not dispense or accept the medication. You are provided with 8 potential reasons why you would or would not dispense or accept the medication. Of these 8 reasons, please rank the top 4 reasons why you made that choice - with " 1 " being the primary reason.

Scenario 1: Placebo use in patient care

While on rounds, the doctor mentions to his students and interns that he plans to put Mrs. Aisha on placebo capsules to see if her symptoms are real. He explains to the students that placebos have been used in patient care for decades because research has shown that a placebo effect exists. A placebo effect is a physiologic response to treatment even though no active medication is used.

As the pharmacist, would you dispense the placebo capsules?

$\begin{array}{ll}\text { a. Yes, I would dispense the placebo } & \text { [ ] } \\ \text { b. No, I would not dispense the placebo }\end{array}$ 
From the list of possible reasons provided, please rank the top 4 reasons why you would or would not dispense the placebo capsules

- The patient has a right to make an informed and voluntary decision [ ]

— The pharmacist has a right to make an informed and voluntary decision [ ]

It is the pharmacist's responsibility to not intentionally create harm or injury [ ]

_ It is the pharmacist's responsibility to act on behalf of the patient

_ The pharmacist must be truthful

_ All patients should receive equal treatment

The pharmacist must maintain the pharmacist-physician relationship [ ] ]

The pharmacist must maintain the pharmacist-patient relationship

Scenario 2: Sedating unruly patient

Mr. Ibrahim is a 45 year old patient admitted to the hospital for a psychiatric evaluation. He is incoherent and shouting at passersby. The doctor does not believe that Mr. Ibrahim poses any physical danger to himself or others but decides to prescribe sedatives.

Would you dispense the sedative?

a. Yes, I would dispense the sedative

b. No, I would not dispense the sedative

From the list of possible reasons provided, please rank the top 4 reasons why you would or would not dispense the sedative:

- The patient has a right to make an informed and voluntary decision [ ]

- The pharmacist has a right to make an informed and voluntary decision [ ]

_ It is the pharmacist's responsibility to not intentionally create harm or injury [ ]

_ It is the pharmacist's responsibility to act on behalf of the patient

- The pharmacist must be truthful

All patients should receive equal treatment

The pharmacist must maintain the pharmacist-patient relationship

Scenario 3: Assisted suicide

Mrs. Zainab is a 50 year old patient with advanced AIDS. Due to the compromised immune system, she only has a few days to weeks left to live and is in terrible pain. She does not want to suffer through a painful death and wishes to end her life now. She asks the doctor to provide her with an overdose of morphine to hasten her death.

Would you dispense the morphine?

a. Yes, I would dispense the morphine

b. No, I would not dispense the morphine

From the list of possible reasons provided, please rank the top 4 reasons why you would or would not dispense the morphine:

— The patient has a right to make an informed and voluntary decision [ ]

_ The pharmacist has a right to make an informed and voluntary decision [ ]

_ It is the pharmacist's responsibility to not intentionally create harm or injury [ ]

_ It is the pharmacist's responsibility to act on behalf of the patient

The pharmacist must be truthful

_ All patients should receive equal treatment

- The pharmacist must maintain the pharmacist-physician relationship

- The pharmacist must maintain the pharmacist-patient relationship

Scenario 4: Expired medications for mission trip

Mr. Musa, a clinical pharmacist, heading on medical mission trip to

Konduga. The non-profit group which organizes these mission trips does not have funds available to purchase medications and medical supplies. A drug representative offers Mr. Musa a large supply of expired medications and supplies for the trip.

If you were Mr. Musa, would you accept the expired medications for use on the mission trip?

a. Yes, I would accept the expired medications

b. No, I would not accept the expired medications

From the list of possible reasons provided please rank the top 4 reasons why you would or would not accept the expired medications:

- The patient has a right to make an informed and voluntary decision

The pharmacist has a right to make an informed and voluntary decision [ ]

_ It is the pharmacist's responsibility to not intentionally create harm or injury [ ]

_ It is the pharmacist's responsibility to act on behalf of the patient

The pharmacist must be truthful

All patients should receive equal treatment

The pharmacist must maintain the pharmacist-physician relationship

_ The pharmacist must maintain the pharmacist-patient relationship

Adapted from: Smith \& Dinkins (2015) \& Schlesselman (2014) 